\section{Measurement and modeling of effective cable parameters of unshielded conductors}

\author{
Karl Hollaus
}

Institute for Analysis and Scientific Computing, Technische Universitat Wien, Wien, Austria and Silicon Austria Labs GmbH, Graz, Austria

Susanne Bauer

IGTE, TU Graz, Graz, Austria

Michael Leumüller

Technische Universität Wien, Wien, Austria, and

Christian Türk

BMLV, Wien, Austria
Cable parameters of unshielded conductors

Received 14 March 2021 Revised 30 November 2021 Accepted 15 December 2021

\begin{abstract}
Purpose - Cables are ubiquitous in electronic-based systems. Electromagnetic emission of cables and crosstalk between wires is an important issue in electromagnetic compatibility and is to be minimized in the design phase. To facilitate the design, models of different complexity and accuracy, for instance, circuit models or finite element (FE) simulations, are used. The purpose of this study is to compare transmission line parameters obtained by measurements and simulations.

Design/methodology/approach - Transmission line parameters were determined by means of measurements in the frequency and time domain and by $\mathrm{FE}$ simulations in the frequency domain and compared. Finally, a Spice simulation with lumped elements was performed.

Findings - The determination of the effective permittivity of insulated wires seems to be a key issue in comparing measurements and simulations.

Originality/value - A space decomposition technique for a guided wave on an infinite configuration with constant cross-section has been introduced, where an analytic representation in the direction of propagation is used, and the transversal fields are approximated by FEs.
\end{abstract}

Keywords Time-domain-reflectometry (TDR), Numerical simulation, Cable parameter extraction, Finite element method (FEM), Spice, Circuit analysis, S-parameter analysis

Paper type Research paper

\section{Introduction}

One main goal of the present work is to match measurement data, Spice (LTSpice, 2020) and finite element (FE) simulations to create models of different complexity. A configuration as simple as a single insulated wire above ground needs to be modeled, built and tested to

(C) Karl Hollaus, Susanne Bauer, Michael Leumüller and Christian Türk. Published by Emerald Publishing Limited. This article is published under the Creative Commons Attribution (CC BY 4.0) licence. Anyone may reproduce, distribute, translate and create derivative works of this article (for both commercial and non-commercial purposes), subject to full attribution to the original publication and authors. The full terms of this licence may be seen at http://creativecommons.org/licences/by/4.0/legalcode

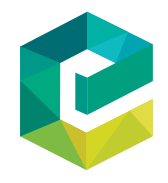

COMPEL - The international journal for computation and mathematics in electrical an electronic engineering Vol. 41 No 3,2022 1. 41 No. 3, 2022 pp. 1041-1051
Emerald Publishing Limited
$0332-1649$ DOI 10.1108/COMPEL-03-2021-0098 
COMPEL 41,3 extract the equivalent RLGC-parameters, characteristic impedance $Z_{0}$, velocity factor $v_{f}$, as well as delay time $t_{d}$. Naturally, simulation data and measurement data should match, but due to imperfections of the physical test setup, such as connectors, stray capacitances, imperfect return conductors (e.g. copper ground plane), measurement inaccuracies, imperfect terminations and models for simulation that use a $0.5 \mathrm{~mm}$ copper plane for current return, have no connectors and stray effects involved, this is not easy to accomplish. Theory and exemplary simulation data of micro-strips of a printed circuit board (PCB) can be found in Hollaus et al. (2008), Caniggia and Maradei (2008), Chaturvedi et al. (2016) as well as in Eisenstadt and Eo (1992). Careful consideration of parasitic effects is necessary, and concise analysis of their order is a prerequisite to obtaining reliable results. Because the setup here appears to be simple, it serves as a basis for more complex models which involve coupling effects between high impedance traces or low impedance loops on a PCB. Measured data and simulation, as well as underlying models, still need to match.

\section{Measurement setups}

Each test setup requires a specific configuration and termination. Apart from the Spice simulation (LTSpice, 2020), the setups use either a polyvinyl chloride (PVC) or silicone insulated single wire above a copper ground plane (1.0 m times $1.0 \mathrm{~m}$ and $0.5 \mathrm{~mm}$ thick), which can be considered infinite. The PVC insulated wires have a copper-conductor of $1.5 \mathrm{~mm}^{2}$ and red and black insulation with a diameter of $3.0 \mathrm{~mm}$. The silicone insulated wires have a copper conductor of $1.0 \mathrm{~mm}^{2}$ and red and black insulation with a diameter of $2.3 \mathrm{~mm}$. All four wires are $1.0 \mathrm{~m}$ long. The wires are fixed to the ground plane with adhesive tape to minimize the distance between insulation and copper - there should ideally be no air gap between cable and ground plane. The test equipment is connected via SMA-connectors with their ground terminals soldered to the ground plane and the inner terminal to the cable under test (CUT).

\subsection{Time domain reflectometry}

The time domain reflectometry (TDR)-test uses an HP8753D $6 \mathrm{GHz}$ vector network analyzer (VNA) which subsequently converts the acquired response into the time domain by means of an inverse fast Fourier transform. The calibration requires a test cable that is terminated with the system impedance of $50 \Omega$, an open and a short standard. The test requires the far end to be open to provide a quasi-full positive reflection of the incident signal. The TDR-test set is connected to the near end of the CUT.

\subsection{Frequency domain testing - S-parameters}

The acquisition of the S-parameters uses a Rohde \& Schwarz ZVL6 as VNA. The calibration requires a full two-port through-open-short-match procedure. The measurement requires the CUT to be connected to both ports of the VNA. It represents a fully terminated CUT.

All data from the VNA consist of input reflection coefficient $S_{11}$, forward transmission gain $S_{21}$, reverse transmission gain $S_{12}$ and output reflection coefficient $S_{22}$ with magnitude in $d B$ and the respective phases in degrees. A conversion to a linear scale and into radians [as in Tuerk et al. (2020)] needs to be accomplished before application of the following set of equations (1) and (2).

With $Z_{l}$ being the characteristic impedance of the CUT 
and the propagation constant $\gamma$ in

$$
Z_{l}=\sqrt{Z_{0}^{2} \cdot \frac{\left(1+S_{11}\right)^{2}-S_{21}^{2}}{\left(1-S_{11}\right)^{2}-S_{21}^{2}}}
$$

Cable

$$
e^{-\gamma l}=\left(\frac{1-S_{11}^{2}+S_{21}^{2}}{2 S_{21}} \pm K\right)^{-1}
$$

with $l$ being the length of the CUT and $K$ given by the following

$$
K=\sqrt{\frac{\left(1+S_{11}^{2}-S_{21}\right)^{2}-\left(2 S_{11}\right)^{2}}{\left(2 S_{21}\right)^{2}}}
$$

the per unit length (PUL) parameters of the CUT can be extracted from the S-parameters.

Considering ambiguities, the $\pm K$-term must be accounted for in the following extraction of the RLGC-parameter set

$$
\begin{aligned}
R^{\prime} & =\operatorname{Re}\left(\gamma Z_{l}\right), \\
L^{\prime} & =\frac{\operatorname{Im}\left(\gamma Z_{l}\right)}{\omega}, \\
G^{\prime} & =\operatorname{Re}\left(\frac{\gamma}{Z_{l}}\right) \text { and } \\
C^{\prime} & =\frac{\operatorname{Im}\left(\frac{\gamma}{Z_{l}}\right)}{\omega},
\end{aligned}
$$

respectively.

\section{Simulation methods}

\subsection{Spice simulation}

Because the CUT is a sample of $1.0 \mathrm{~m}$ in length and the maximum frequency is $500 \mathrm{MHz}$ in the case of the S-parameter measurement, the simulation model has been split into pieces of $0.1 \mathrm{~m}$ for the purpose of the simulation. The PUL data acquired are divided by 10 as far as $L^{\prime}$ and $R^{\prime}$ are concerned, $C^{\prime}$ and $G^{\prime}$ are multiplied by 10 . In total, the CUT in the simulation represents $1.0 \mathrm{~m}$, and the condition of segments being significantly shorter than $\lambda / 10$ is still satisfied.

The model in Figure 1 shows 10 sections, each of them with 4 lumped elements ( $R 1, L 1$, $C 1, G 1$ to $R 10, L 10, G 10, C 10)$, the termination of the model represented by $R 11$ and the respective parameters which are applied to all elements. To get S-parameters from the schematic, the directive . net I (R11) V1 is used. This parameter set provides the result given in section 4 in Figure 6.

\subsection{Finite element method}

The benchmark with details is presented in Figure 2 and consists of a PVC or silicon insulated copper wire above a copper plate. The length of the wire has been selected to be 
COMPEL

41,3

1044

Figure 1.

Discrete Model in LTSpice®

\section{Figure 2.}

Insulated copper wire above ground, not to scale
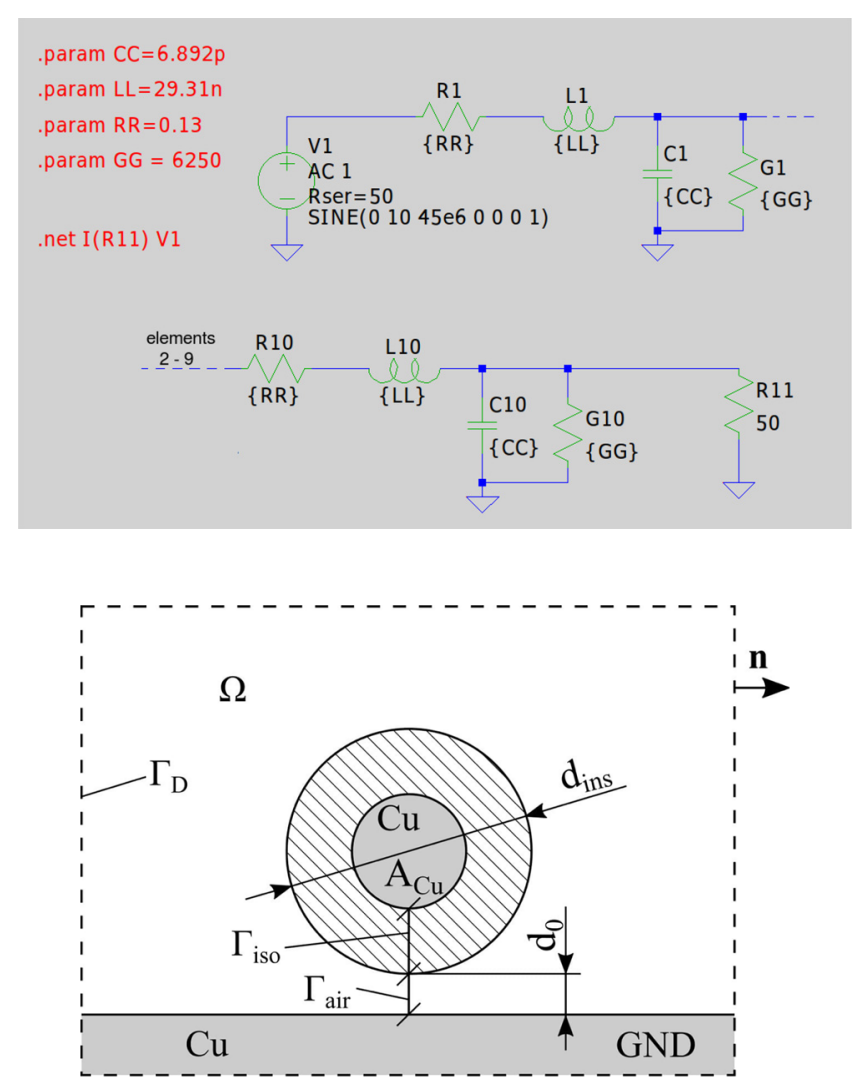

Notes: PVC: cross section $A_{C u}=1.5 \mathrm{~mm}^{2}$,

diameter of insulation $d_{i n s}=3.0 \mathrm{~mm}$ and air gap $d_{0}=0.1 \mathrm{~mm}$. Silicon: $A_{C u}=1.0 \mathrm{~mm}^{2}, d_{i n s}=2.3$ $\mathrm{mm}$ and $d_{0}=0.1 \mathrm{~mm}$

$1.0 \mathrm{~m}$, and the copper plate with a thickness of $0.5 \mathrm{~mm}$ is assumed to have infinite dimensions. The air gap represents an estimated average in contrast to the physical setup of the measurements.

The basic boundary value problem to be solved in the frequency domain reads

$$
\begin{array}{rlrl}
\operatorname{curl} \mu^{-1} \operatorname{curl} \boldsymbol{A}+j \omega(\sigma+j \omega \varepsilon) \boldsymbol{A} & =0 & & \text { in } \Omega, \\
\boldsymbol{A} \times \boldsymbol{n}=\boldsymbol{\alpha} & & \text { on } \Gamma_{D},
\end{array}
$$

where $A$ is the magnetic vector potential (MVP), $\mu$ the magnetic permeability, $\sigma$ the electric conductivity, $\varepsilon$ the electric permittivity, $j$ the imaginary unit, $\omega$ the angular frequency, $\boldsymbol{\alpha}$ some Dirichlet boundary condition, $\Omega$ the problem domain, $\Gamma_{D}$ the Dirichlet boundary and $\boldsymbol{n}$ the unit normal vector, see Figure 2 . The domain $\Omega$ comprises air, copper wire, insulation and GND, respectively, where $\Gamma_{D}$ is its outer boundary. The following considerations for the FE simulations are based on a Cartesian coordinate system. The 
transversal fields are lying in the drawing plane, compare with Figure 2. The direction of propagation corresponds to the $z$-coordinate. The problem for the $\mathrm{FE}$ simulations is assumed to be infinitely long in the direction of propagation. To take into account the specific nature of the present problem, the MVP has been decomposed into two components, a transversal component $\mathbf{A}_{\tau}(x, y)$ lying in the drawing plane and $A_{z}(x, y)$, which points into the direction of propagation

$$
\boldsymbol{A}=\left(\boldsymbol{A}_{\tau}(x, y), A_{z}(x, y)\right)^{T} e^{-\gamma z},
$$

where $\gamma=\sqrt{j \omega \mu(\sigma+j \omega \varepsilon)}$ with $A_{\tau, h} \in H\left(\operatorname{curl}, \Omega_{2 D}\right)$ and $A_{z, h} \in H^{1}\left(\Omega_{2 D}\right)$ for the FE approximation indicated by the additional index $h$, see Schöberl and Zaglmayr(2005). Because the dependence of $A$ in equation (5) with respect to $z$ is known, a two-dimensional FE model suffices. The lumped elements $R^{\prime}, C^{\prime}$, etc., have been calculated based on the corresponding losses and energies PUL in the electromagnetic field. For instance, $C^{\prime}$ has been determined with the electric energy $W_{e, \tau}^{\prime}$ due to the transverse electric field $\mathbf{E}_{\tau}$, which is shown in Figure 7, and $R^{\prime}$ with the aid of the losses $P_{z}^{\prime}$ caused by currents due to the electric field component $\mathbf{E}_{z}$. The lumped elements are obtained by

$$
\begin{aligned}
R^{\prime} & =\frac{2 P_{z}^{\prime}}{I^{2}}, \\
L^{\prime} & =\frac{2 W_{m, z}^{\prime}}{I^{2}}, \\
G^{\prime} & =\frac{2 P_{\tau}^{\prime}}{U^{2}} \text { and } \\
C^{\prime} & =\frac{2 W_{e, \tau}^{\prime}}{U^{2}},
\end{aligned}
$$

respectively. The voltage $U$ between the copper wire and ground plate is prescribed to excite the problem; the current $I$ is obtained by means of simulation. The $z$-components of the current densities in the wire and in the ground plate are opposed. The current in the copper wire should theoretically match the current in the GND plane except for the sign. The crosssections of the copper wire and of the GND-plane are completely different; consequently, their FE-meshes too. Therefore, one cannot expect an exact match of the two currents because of numerical inaccuracies. For instance, prescribing $U=1 \mathrm{~V}$ yields, $I=-0.01411-$ $j 0.00013 \mathrm{~A}$ in the wire and $I=0.01461+j 0.00061 \mathrm{~A}$ in the GND-plane, which is satisfactorily accurate.

\section{Results}

All data shown were acquired with CUTs $1.0 \mathrm{~m}$ in length or referred to as $1.0 \mathrm{~m}$ as far as simulation data are concerned.

\subsection{Frequency domain data}

The measurement in the frequency domain was performed using a 2-port VNA (ZVL6). Both ends of the CUT are connected to the VNA, which represents a termination with $50 \Omega$ at either end. The obtained set of S-parameters was subsequently converted into RLGC-data. The results with the given PVC-insulated conductor can be seen in Table 1. 
COMPEL 41,3

\section{6}

Measurements of a red and black silicone-insulated conductor show a similar picture, and the obtained results are given in Table 2.

It is important to note that the conductors with different colored insulators exhibit differing RLGC parameters. This will also be addressed in Section 4.2.

The data extraction requires some arithmetic: The test point chosen is the lowest frequency minimum of the imaginary part of $Z_{S 11}$, the impedance of the CUT derived from $S_{11}$, as can be seen in Figure 3 for the conductor with the black PVC insulator.

The impedance seen at Port 1 of the VNA equals

\begin{tabular}{llccc}
\cline { 2 - 4 } & Parameter & PVC black & PVC red & Unit \\
\cline { 2 - 4 } & $f$ & 59.46036 & 57.46873 & $\mathrm{MHz}$ \\
& $Z_{l}$ & 65.21131 & 63.88990 & $\Omega$ \\
& $R$ & 1.325222 & 4.935247 & $\Omega$ \\
& $L$ & 293.0912 & 285.8608 & $\mathrm{nH}$ \\
& $\mathrm{C}$ & 68.92185 & 70.03098 & $\mathrm{pF}$ \\
Table 1. & $\mathrm{G}$ & 1068.469 & 704.8839 & $\mu \mathrm{S}$ \\
Frequency domain, & $v_{p}$ & $2.224949 \cdot 10^{8}$ & $2.235000 \cdot 10^{8}$ & $\mathrm{~m} / \mathrm{s}$ \\
PVC & $t_{d}$ & 0.7416498 & 0.7450001 & 1 \\
& $\varepsilon_{r, e f f}$ & 4.494484 & 4.474273 & $\mathrm{~ns}$ \\
\hline
\end{tabular}

Table 2.

Frequency domain, silicone

\begin{tabular}{lccc}
\hline Parameter & Silicone black & Silicone red & Unit \\
\hline$f$ & 59.33388 & 55.78086 & $\mathrm{MHz}$ \\
$Z_{l}$ & 70.32518 & 69.65358 & $\Omega$ \\
$R$ & 2.415429 & 1.786379 & $\Omega$ \\
$L$ & 307.7858 & 305.5429 & $\mathrm{nH}$ \\
$C$ & 62.23388 & 62.97747 & $\mathrm{pF}$ \\
$G$ & 639.8732 & 284.2796 & $\mu \mathrm{S}$ \\
$v_{p}$ & $2.284874 \cdot 10^{8}$ & $2.279667 \cdot 10^{8}$ & $\mathrm{~m} / \mathrm{s}$ \\
$v_{f}$ & 0.7616247 & 0.7598888 & $\mathrm{~ns}$ \\
$t_{d}$ & 4.376609 & 4.386607 & 1 \\
$\varepsilon_{r, \text { eff }}$ & 1.723923 & 1.731809 & \\
\hline
\end{tabular}

Figure 3.

$Z_{S 11}$, real and imaginary part

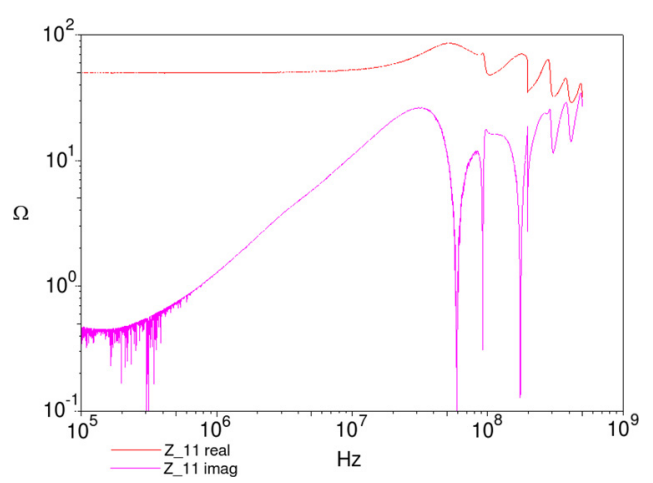




$$
Z_{S 11}=Z_{0} \cdot \frac{1+S_{11}}{1-S_{11}}
$$

and considers the magnitude and phase of the real and imaginary parts of $Z_{S 11}$.

\subsection{Time domain data}

As mentioned above, the TDR test set is used to acquire the "flight time" of a pulse incident on the CUT. The effective permittivity $\varepsilon_{r, \text { eff }}$ can directly be derived from the time delay $t_{d}=t_{\text {flight }} / 2$ as given by the TDR test set using

$$
\varepsilon_{r, e f f}=\left(\frac{1}{v_{f}}\right)^{2}
$$

with $v_{f}$ being the velocity factor of the wave on the CUT, which is always $\leq 1$, and

$$
v_{f}=\frac{1}{c_{0} \cdot t_{d}} \cdot l
$$

with $c_{0}$ being the speed of light in a vacuum and $l$ being the length of the CUT.

Tables 3 and 4 show the parameter "unit" which has not been explained so far. TDR measurements usually normalize the amplitude of the reflected wave to the system impedance. A full reflection from an open terminated cable is then represented by a unit of 2 , whereas no reflection at the end of a cable, i.e. a proper termination with the impedance of the conductor, will show a unit of 1 .

A fraction or a multiple of a unit is consequently a measure of the impedance of the cable, as outlined in Figure 4.

\begin{tabular}{|c|c|c|c|c|}
\hline Parameter & PVC black & PVC red & Unit & \\
\hline Flight time & 9.732 & 9.568 & ns & \\
\hline$t_{d}$ & 4.866 & 4.784 & ns & \\
\hline$v_{f}$ & 0.6855 & 0.6973 & 1 & \\
\hline$\varepsilon_{r, e f f}$ & 2.128 & 2.057 & 1 & \\
\hline$r_{f}$ & 0.145 & 0.136 & 1 & Table 3. \\
\hline$Z_{1}$ & 66.95 & 65.74 & $\Omega$ & Time domain, $\mathrm{PVC}$ \\
\hline
\end{tabular}

The "flight time" mentioned earlier is the time a pulse incident into the CUT takes until it returns from the open end of the CUT. The pulse "travels" two times the cable

\begin{tabular}{lcccc}
\hline Parameter & Silicone black & Silicone red & Unit & \\
\hline Flight time & 9.78 & 9.624 & $\mathrm{~ns}$ & \\
$t_{d}$ & 4.89 & 4.812 & $\mathrm{~ns}$ & \\
$v_{f}$ & 0.6821 & 0.6927 & 1 & Table 4. \\
$\varepsilon_{r, \text { eff }}$ & 2.149 & 2.0839 & 1 & Time domain, \\
$r_{f}$ & 0.189 & 0.187 & 1 & silicone \\
$Z_{l}$ & 73.30 & 73.00 & & \\
\hline
\end{tabular}


COMPEL

41,3

\section{8}

Figure 4.

Parameters of a TDRresponse

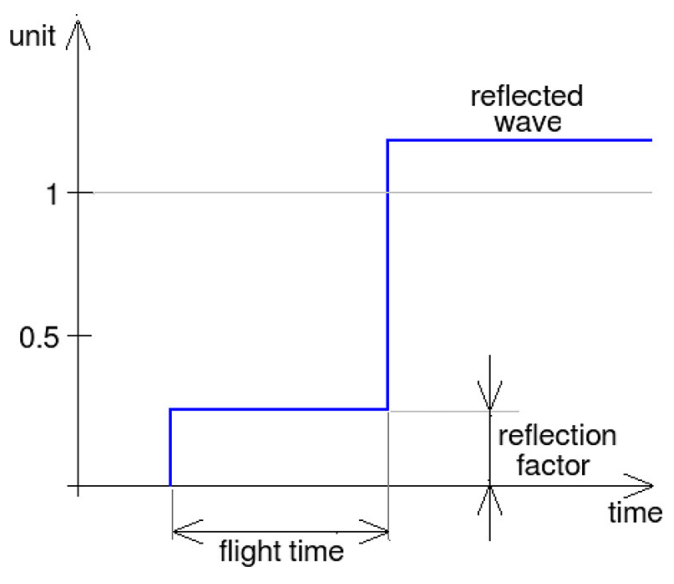

length; therefore, the delay time $t_{d}$ is half the "flight time". The "reflection factor" mentioned in Figure 4 can be converted into an impedance value of the CUT by means of the following equation

$$
Z_{l}=Z_{0} \cdot \frac{1+r_{f}}{1-r_{f}}
$$

where $Z_{l}$ represents the characteristic impedance of the CUT and $r_{f}$ the reflection factor with a sign.

A representative measurement is shown in Figure 5.

\subsection{Spice results}

To verify the applicability of the model data and their match to measured results, the acquired cable data of "PVC black" have been used. It is clear that the idealized simulation because no parasitics are considered, shows minor deviations. Yet, a first verification with the resonant frequency shows a satisfying match, as given in Figure 6.

The trace shows $Z_{S 11}$ derived from $S_{11}$ using equation (7) but a blank and the resonance at $66.14 \mathrm{MHz}$, which is a close match to the measurement results.
Figure 5.

Exemplary TDRpattern

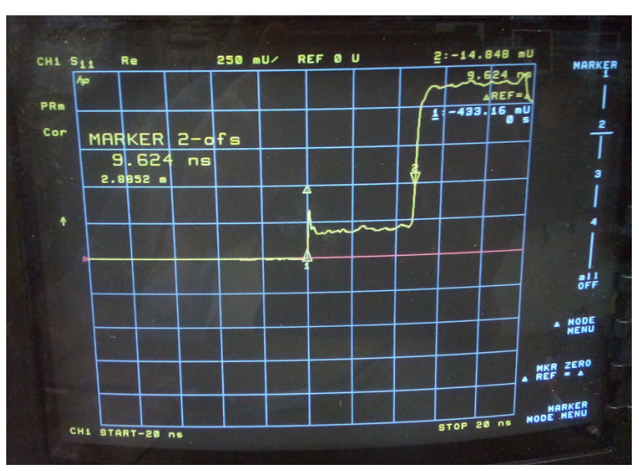




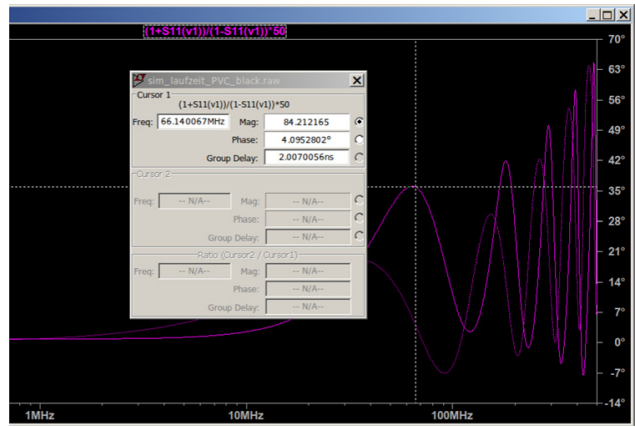

Cable parameters of unshielded conductors

1049

Figure 6.

Resonance match for PVC black

\subsection{Finite element simulations}

Homogenous Dirichlet boundary conditions $\mathbf{A}_{\tau} \times \mathbf{n}=\mathbf{0}$ and $A_{z}=0$ are selected on $\Gamma_{D}$. The shortest line from the copper wire to GND consists of $\Gamma_{i s o}$ and $\Gamma_{\text {air }}$, compared with Figure 2 . A voltage of $U=1 \mathrm{~V}$ as excitation is prescribed by the tangential component of $\mathbf{A}_{\tau}$ along $\Gamma_{i s o}$ and $\Gamma_{\text {air }}$ corresponding to $\epsilon_{r}$ of air and insulator and the lengths of $\Gamma_{i s o}$ and $\Gamma_{\text {air }}$, respectively.

Simulations have been carried out with Netgen/NGSolve (Schöberl, 2021). The transversal electric field is obtained by

$$
E_{\tau}=-j \omega A_{\tau},
$$

a field plot is presented in Figure 7. The measurement and simulation results are summarized in Table 5. No losses have been considered in the insulation; $G^{\prime}$ solely takes

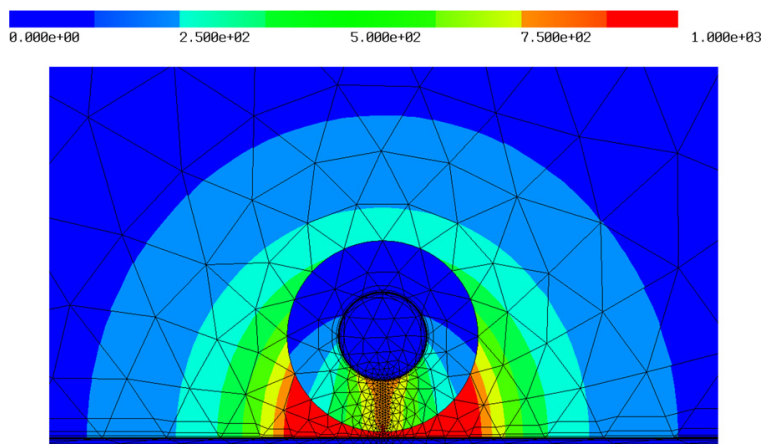

Figure 7.

Transverse electric field $\left|\mathbf{E}_{\tau}\right|$ for the PVC insulated wire, detail.

The FE model

consists of 13,160

elements

\begin{tabular}{|c|c|c|c|c|c|c|}
\hline \multirow[b]{2}{*}{ Parameter } & \multirow[b]{2}{*}{ Unit } & \multicolumn{2}{|c|}{ Measurement } & \multicolumn{2}{|c|}{ Simulation } & \\
\hline & & PVC & Silicone & PVC & Silicone & \\
\hline$f$ & $\mathrm{MHz}$ & 59.3 & 59.5 & 59.3 & 59.5 & \\
\hline$R^{\prime}$ & $\Omega / \mathrm{m}$ & 1.33 & 2.42 & 1.19 & 1.45 & \\
\hline$L^{\prime}$ & $\mathrm{nH} / \mathrm{m}$ & 293 & 308 & 306 & 293 & \\
\hline$C^{\prime}$ & $\mathrm{pF} / \mathrm{m}$ & 68.9 & 62.2 & 81.8 & 72.9 & Table 5. \\
\hline$G^{\prime}$ & $\mathrm{mS} / \mathrm{m}$ & 1.07 & 0.64 & 0.12 & 0.14 & Measurement and \\
\hline$Z_{l}$ & $\Omega$ & 65.2 & 70.3 & 61.1 & 63.4 & simulation results \\
\hline
\end{tabular}


COMPEL into account losses due to the transverse field in copper, which explains the large 41,3 difference between measurement and simulation. The true values of several parameters are not known. To study the influence on transmission line parameters (TLPs), several simulations have been carried out. The uncertain parameters have been varied in a meaningful and feasible range. Results are presented in Tables 6-8. The influence of $\varepsilon_{r}$ on the TLPs is big, whereas that of $d_{0}$ and $d_{i n s}$ is rather moderate. However, it is worth mention that for a very small $d_{0}$ and a feasible $\mathrm{FE}$ mesh, the limit value $C^{\prime}=93.5 \mathrm{pF}$ has been found.

\section{Conclusions}

Measurements in the frequency domain and in the time domain show a reasonably accurate match of the results.

It needs to be said that both approaches complement and confirm each other. The FD testing requires a terminated line (with the system impedance), the TD testing needs an open line end, i.e. without termination matching the system impedance.

Future work will extend the model to extract estimated emissions from a single conductor as well as bus structures at frequencies of interest. This will be examined with and without a grounded plane with subsequent verification and matching to get reliable simulation models. Three-dimensional FE models will be used to appropriately simulate relevant emissions. The simulation models shall be optimized for fast and reliable

Table 6.

Relative permittivity of the insulator

\begin{tabular}{lccccc}
\hline$\varepsilon_{r}$ & $R^{\prime}$ & $L^{\prime}$ & $C^{\prime}$ & $G^{\prime}$ & $Z_{l}$ \\
1 & $\Omega$ & $\mathrm{nH} / \mathrm{m}$ & $\mathrm{pF} / \mathrm{m}$ & $\mathrm{mS} / \mathrm{m}$ & $\Omega$ \\
\hline 3 & 1.15 & 302 & 71.0 & 0.11 & 65.2 \\
4 & 1.19 & 306 & 81.8 & 0.12 & 61.1 \\
5 & 1.22 & 309 & 90.5 & 0.12 & 58.4 \\
\hline
\end{tabular}

Table 7.

Air gap width

\begin{tabular}{lccccc}
\hline$d_{0}$ & $R^{\prime}$ & $L^{\prime}$ & $C^{\prime}$ & $G^{\prime}$ & $Z_{l}$ \\
$\mathrm{~mm}$ & $\Omega$ & $\mathrm{nH} / \mathrm{m}$ & $\mathrm{pF} / \mathrm{m}$ & $\mathrm{mS} / \mathrm{m}$ & $\Omega$ \\
\hline 0.08 & 1.21 & 304 & 84.4 & 0.13 & 60.1 \\
0.1 & 1.19 & 306 & 81.8 & 0.12 & 61.1 \\
0.12 & 1.17 & 307 & 79.5 & 0.11 & 62.2 \\
\hline
\end{tabular}

Table 8.

Diameter of the insulator

\begin{tabular}{lccccc}
\hline$d_{\text {ins }}$ & $R^{\prime}$ & $L^{\prime}$ & $C^{\prime}$ & $G^{\prime}$ & $Z_{l}$ \\
$\mathrm{~mm}$ & $\Omega$ & $\mathrm{nH} / \mathrm{m}$ & $\mathrm{pF} / \mathrm{m}$ & $\mathrm{mS} / \mathrm{m}$ & $\Omega$ \\
\hline 2.9 & 1.21 & 298 & 82.7 & 0.12 & 60.1 \\
3.0 & 1.19 & 306 & 81.8 & 0.12 & 61.1 \\
3.1 & 1.17 & 313 & 81.0 & 0.11 & 62.2 \\
\hline
\end{tabular}


simulations based on a sensitivity analysis showing the impact of parameter variations like $d_{0}$ in Figure 2.

\section{References}

Caniggia, S. and Maradei, F. (2008), Signal Integrity and Radiated Emission, Wiley, ISBN 978-0-470-51166-4.

Chaturvedi, S., Božanić, M. and Sinha, S. (2016), "Extraction of transmission line parameters and effect of conductive substrates on their characteristics", Romanian Journal of Information, Science and conductors Technology, Vol. 19 No. 3, pp. 199-212.

Eisenstadt, W.R. and Eo, Y. (1992), "S-parameter-based IC interconnect transmission line characterization", IEEE Transactions on Components, Hybrids, and Manufacturing Technology, Vol. 15 No. 4, pp. 483-490.

Hollaus, K., Bíró, O., Caldera, P., Matzenauer, G., Paoli, G. and Plieschenegger, G. (2008), "Simulation of crosstalk on printed circuit boards by FDTD, FEM, and a circuit model", IEEE Transactions on Magnetics, Vol. 44 No. 6, pp. 1486-1489.

LTSpice (2020), "SPICE simulation software, analog devices", available at: https://tspice.analog.com/ software/LTspiceXVII.exe (accessed October 2021).

Schöberl, J. (2021), "Netgen/NGSolve”, available at: https://ngsolve.org (accessed October 2021).

Schöberl, J. and Zaglmayr, S. (2005), "High order nédélec elements with local complete sequence properties", COMPEL - The International Journal for Computation and Mathematics in Electrical and Electronic Engineering, Vol. 24 No. 2, pp. 374-384.

Tuerk, C., Pommerenke, D. and Bauer, S. (2020), "Improved alternative method for fast and simple transfer impedance measurements", Letters on Electromagnetic Compatibility Practice and Applications, Vol. 2 No. 4, doi: 10.1109/LEMCPA.2020.3024810.

\section{Corresponding author}

Karl Hollaus can be contacted at: karl.hollaus@tuwien.ac.at

For instructions on how to order reprints of this article, please visit our website: 